\title{
LE PaQUet DE PANSEMENT DANS LES DIFFÉRENTES NATIONS
}

Nous avons indiqué, d'après les articles de M. Laval dans le Caducée ${ }^{1}$, la composition du paquet de pansement de quelques armées. Si nous revenons en deux mots sur ce sujet, c'est pour mentionner un mémoire très complet sur ce sujet de Oberstabsarzt $\mathrm{D}^{r}$ Blau2. Pour rendre les données fournies plus uniformes, l'auteur a établi un tableau comparatif détaillé comportant la désignation, la forme, le mode de paquetage, le contenu, le mode d'emploi et le mode de transport de tous les sachets de pansements d'armée connus, soit vingt pansements différents.

Contentons-nous de signaler quelques-unes des observations qui suivent ce tableau : ainsi l'auteur loue, entre autres, l'emploi de trois langues dans les indications que porte le paquet suisse sur l'emploi du pansement par le blessé; cette mesure qui, dans l'espèce, répond au besoin des trois différentes populations de la Suisse, est, d'autre part, tout à fait dans l'esprit de la Convention de Genève, en tant qu'utile aussi pour les blessés de l'armée ennemie; cet exemple serait donc bon à imiter.

L'auteur fait ressortir, d'autre part, l'avantage du système allemand qui comporte deux petits paquets au lieu d'un seul, le second pansement pouvant être utilisé séparérnent sans avoir été souillé.

En Allemagne, tous les militaires portent le sachet: soldats, brancardiers, officiers de la troupe, officiers sanitaires, employés, etc.; dans d'autres armées, il n'est porté que par le soldat.

L'indication du mode d'emploi sur l'extérieur du paquel, en usage dans quelques armées, présente le grand avantage de pouvoir être consulté à loisir et permettre ainsi une familiarisation plus grande de ce texte pour le soldat. En Russie et dans les Pays-Bas, ce texte est reproduit de nouveau a l'intérieur du paquet, mesure utile pour le cas où l'indication extérieure se trouverait effacée ou ternie par l'usure de l'enveloppe.

- Voir T. XXXIV, p. 206. T. XXXVIII, p. 273. T. XXXIX, p. 106.

2 Deutsche militärärztliche Zeitschrift, 1908, $\mathrm{n}^{\circ} 11$, p. 457. 
L'auteur attache peu d'importance à la grandeur du pansement, mais il approuve la fabrication en différentes grandeurs, ainsi que cela se pratique dans la marine du Japon et de l'Allemagne; cela permet de satisfaire aux besoins des plaies par petit calibre ou par projectiles d'artillerie.

Plus importante est la question du poids. La Norvège tient la tête avec des paquets de $21 \mathrm{gr}$. seulement, l'Amérique vient en dernier lieu avec des paquets de $130 \mathrm{gr}$., dépassant de $50 \mathrm{gr}$. les paquets les plus lourds de l'Europe, celui de la marine allemande et celui de la Suisse. Le paquetage joue ici un rôle prépondérant et il va de soi que les enveloppes métalliques sont les plus lourdes, mais aussi les plus hermétiques.

L'auteur fait une critique détaillée du contenu des différents paquets et passe en revue les pièces qui le composent dans chaque pays; c'est là, en effet, le point important de ce pansement, et le problème n'était pas simple à résoudre puisqu'il s'agit de fixer une moyenne pratique répondant le plus possible à tous les cas de blessures par armes à feu. Nous avons eu l'occasion d'aborder la discussion de cette question lorsque nous avons analysé les articles de M. Laval, nous n'y reviendrons donc pas et renvoyons le lecteur sur ce point à l'étude détaillée de $\mathrm{M}$ le $\mathrm{D}^{\wedge}$ Blau.

Pour ce qui regarde la question si discutée de l'asepsie ou de l'antisepsie, l'auteur constate que la grande majorité des armées a adopté le pansement antiseptique; le plus souvent, c'est le sublimé qui a été choisi; en Autriche et en Suisse, on a préféré le vioforme. La méthode combinée, soit imprégnation antiseptique après stérilisation à la vapeur, se pratique en Suisse et en Allemagne; c'est sans doute le procédé le plus logique.

L'enseignement concernant l'emploi du pansement, sa valeur au point de vue de l'évolution subséquente de la plaie, les conditions les plus propres à son efficacité, est de toute importance, et, d'autre part, le point le plus délicat de la question, celui qui a exposé ce mode de pansement au plus grand nombre de critiques. Il est vrai qu'il en est sorti victorieux sur toute la ligne, puisqu'il a été adopté partout, mais cela ne veut pas dire que la question soit résolue et, après l'avoir examinée sous ses différentes faces, l'auteur conclut que le paquet de pansement n'aura toute sa valeur que lorsque son emploi aura été enseigné règlementaire- 
ment aux recrues des différentes armées. Ce vœu, déjà énoncé de différents côtés, entre autres dans une éloquente plaidoirie du $\mathrm{D}^{r}$ Albert Schwarz dans le Militärarst ${ }^{1}$, paraît, en effet, répondre à une indication absolument logique et presque urgente même.

\section{$D^{r}$ Ferrière.}

\section{ALLEMAGNE}

CONFÉrENCE DES COMITÉS DES ASSOGIATIONS RÉGIONALES ALleMANDES DE LA CROIX-ROUGE ${ }^{2}$

Tous les cinq ans, la première fois à Stuttgart, la seconde à Strasbourg, cette année-ci à Dresde du 26-29 mai, les comités des associations régionales allemandes de la Croix-Rouge ont tenu une conférence qui a réuni jusqu'à 108 représentants des différentes sociétés. La présidence était occupée par M. von dem Knesebeck, président du Comité central allemand; le comte de Solms Baruth, commissaire royal et inspecteur militaire de l'assistance volontaire, assistait aux délibérations, ainsi qu'un représentant du Ministère de la guerre prussien.

La conférence se composa de trois jours de délibérations el d'une journée consacrée aux exercices d'une colonne.

Un des sujets les plus importants fut la revision de l'union que les différentes sociétés allemandes, basées sur la Convention de Genève, avaient conclue le 20 avril 1869, et dont le Comité central allemand formait la tête. Cette union s'était réalisée avant même l'unité allemande, et s'était maintenue sans changement malgré les modifications politiques qu'ont subies depuis les Etats allemands, et l'élargissement de la sphère d'action des sociétés ellesmêmes. Une revision était cependant devenue nécessaire. Le projet de revision préparé par la Société wurtembergeoise sut se

$1 \mathrm{~N}^{\circ}$ du 3 lavril 1908, sous le titre de Selbsthilfe.

' J'apres Das Rothe Kreuz n', 72. 